composite should be fairly easy to produce once industry begins the large-scale manufacture of quasicrystal powders used in the material.

\section{Quasicrystals of AINiCo Exhibit Band Structure}

A team of scientists has demonstrated that the electronic states of quasicrystals are more like those of ordinary metals than theorists believed possible. Eli Rotenberg, a staff scientist at the Advanced Light Source (ALS) at Lawrence Berkeley National Laboratory, Karsten Horn of the FritzHaber Institute, Max-Planck Society, Berlin, and their colleagues have found that rather than moving around arbitrarily, electrons in quasicrystals travel in "bands" with distinct momentum and energy. While investigating the electronic structure of a quasicrystalline alloy of aluminumnickel-cobalt (AlNiCo) by means of angleresolved photoemission, the data show that electron momenta and energies are correlated with the structure of the quasicrystal.

As reported in the August 10 issue of Nature, bandlike properties, common in metals and other ordinary crystals, were not expected in quasicrystals. Ordinary metals are good conductors because their valence electrons can move freely from atom to atom; this freedom is facilitated by long-range periodic structure. Since quasicrystals lack periodic structure, theorists expected no such extended electronic states.

"One might imagine that from an electron's point of view the material appears disordered. If so, the electronic states would be confined to localized clusters," Rotenberg said. And theoretical considerations suggested electronic states confined to the quasicrystal's many different local structures.

Rotenberg, Horn, and their colleagues decided to test the prediction with an AlNiCo alloy consisting of stacked planes of atoms exhibiting ten-fold symmetry. By looking at the behavior of electrons in the plane, they could observe the effects of this quasicrystalline ordering; by looking at right angles to the planes, they could observe the effects of the periodic, crystalline-like ordering of the stack.

Peter Gille of the Ludwig-MaximiliansUniversity, Munich, grew the quasicrystal, and the samples were prepared and characterized by Horn and by Wolfgang Theis of the Free University of Berlin. At the ALS, Rotenberg and Horn examined the samples by means of low-energy electron diffraction and by angle-resolved photoemission.

"We measure the emission angles and the kinetic energy of electrons scattered from near the surface of the material by soft x-rays," said Rotenberg. "These are the valence electrons, not as tightly bound as electrons near the atomic cores."

The sample is rotated to get a complete distribution of electron angles and energies. The eventual result is a plot of the electronic states of AlNiCo's valence electrons in "momentum space," the mathematical space in which such fundamental concepts as Fermi surfaces and Brillouin zones are constructed and on which much of the band theory of solids is based.

Rotenberg said, "Our principal findings were that the distribution of the electronic states in momentum space correlates with the electron diffraction pattern, just like in an ordinary crystal. The electrons aren't localized to clusters; instead, they feel the long-range quasicrystal potential."

"We found that the electrons propagate nearly freely, like conduction electrons in an ordinary metal," he continued, "and we found there is a Fermi surface, crossed by nickel and cobalt $d$-electrons. Its topology should determine some of the material's fundamental properties."

\section{Materials with Purple Fluorite Structure may Serve Well in Long- Term Radioactive Waste Container}

In a search for a group of materials that may safely contain radioactive waste for long-term storage, an international team of scientists have found that materials with the purple fluorite structure should hold up extraordinarily well under irradiation. The key seems to be that the atoms in the material's structure are relatively disordered and can shift positions with ease, thereby tolerating minute defects caused by radiation.

For several years, researchers looking for better storage materials than those currently used have directed their studies to a class of materials that belongs to a larger group of ceramics called "complex oxides." The materials in this class share a basic chemical formula: two different pairs of metallic cations and seven oxygen atoms. Depending on their size, the cation pairs may give these materials either a highly ordered or a somewhat disordered structure.

A material akin to the shiny, brown

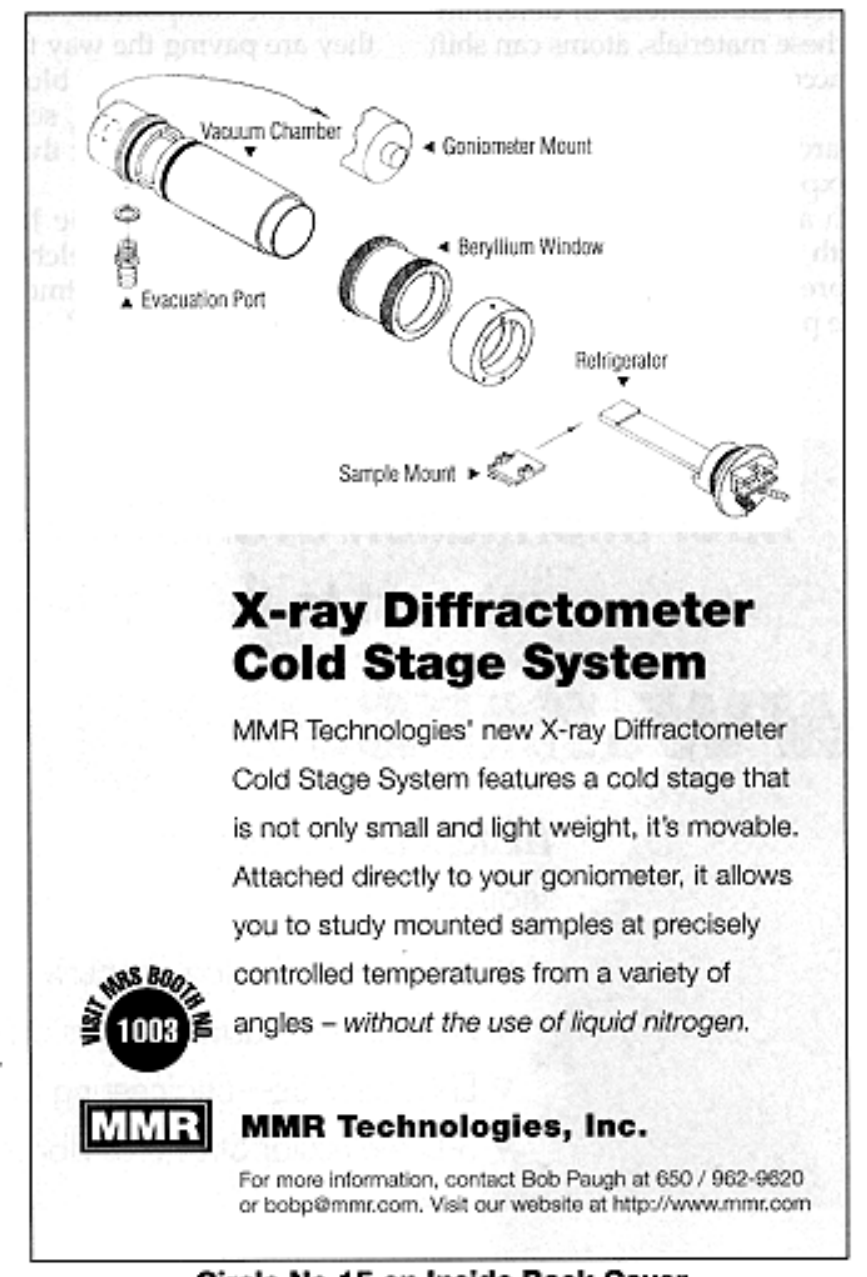

Circle No.15 on Inside Back Cover 